\title{
GROUP A STREPTOCOCCAL MENINGITIS IN A PREVIOUSLY HEALTHY CHILD: A CASE REPORT
}

\section{May AlBarrak}

Division of Infectious Diseases, Department of Pediatrics, Prince Sultan Military Medical City, Riyadh, Saudi Arabia.

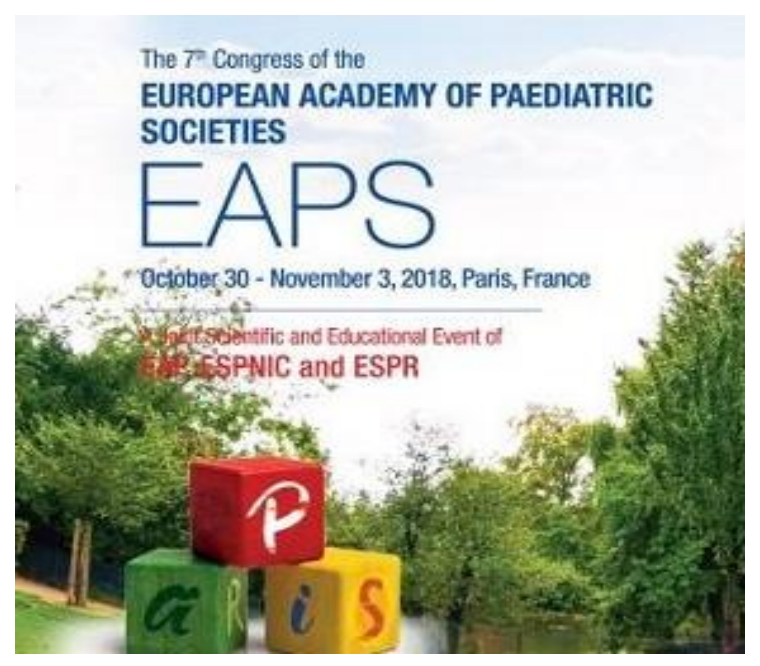

\section{Background}

- Group A streptococcus (GAS) has not been well recognized as cause of meningitis in children. Recent reports have noted great increase in the incidence of invasive GAS infections. Despite of this, meningitis due to GAS is remains rare and usually associated with other focus of infection such as Otitis media, sinusitis and mastoiditis.

\section{Aim}

- To report a rare case of GAS meningitis in a previously healthy child without a clear focus of infection

\section{Case report}

- A previously healthy 4-year-old boy presented with a history of fever, headache and photophobia for the past 24 hours. Central nervous system examination revealed a lethargic child with neck stiffness and positive Brudzinski and Kernig's sign. Other systemic examinations were unremarkable. Lumbar puncture yielded a hazy cerebrospinal fluid (CSF) with high leucocytes count $940 / \mathrm{mm}^{3}$ ( $82 \%$ neutrophils, $4 \%$ lymphocytes and $14 \%$ monocytes), high protein level $1.49 \mathrm{~g} / \mathrm{L}$ and low glucose $1.53 \mathrm{mmol} / \mathrm{L}$. CSF Gram stain showed Gram-positive cocci in chains; subsequently both CSF and blood cultures grew GAS (sensitive to penicillin, ceftriaxone and vancomycin) .Throat swab culture showed no GAS. He was started initially on vancomycin and ceftriaxone. After the culture result, antibiotics were changed to penicillin $G$ high dose $(250,000 \mathrm{iu} / \mathrm{kg} / \mathrm{day})$ and continued for a total of 14 days.

\section{Results}

- His head CT scan was normal and he completely recovered without any neurological sequela

\section{Conclusion}

- GAS meningitis is an unusual manifestation of invasive GAS disease. However, pediatricians should be aware about the possibility of this agent as a cause of meningitis in children even without apparent focus of infection. 